\title{
Enfermedad por citomegalovirus en pacientes receptores de trasplante de corazón en un centro de referencia nacional
}

\author{
Christian Rojas-Contreras, Gabriel De la Cruz-Ku y Bryan Valcarcel-Valdivia
}

\section{Cytomegalovirus disease in post-heart-transplant patients at a national reference center}

Introduction: Heart transplantation remains as the treatment of choice when the heart failure is refractory to the medical or surgical therapy. Therefore, cytomegalovirus disease is an important post-heart-transplant infectious complication. Aims: To describe the prevalence and clinical characteristics of the cytomegalovirus disease after heart transplant surgery. Materials and Methods: A retrospective, descriptive study was conducted. It enrolled 35 heart-transplant patients attended in the Cardiovascular National Institute (INCOR), between 2010 and 2015. The information was obtained through the review of medical records. The demographic and relevant clinical variables were analyzed for the cytomegalovirus disease cases. Results: The population mean age was 39.49 \pm 15.07 years and most of them were male patients (63\%). The prevalence of the cytomegalovirus disease was 5.7\% (two patients), both were seronegative for cytomegalovirus before transplantation. One of the patients had the disease before finishing the valganciclovir prophylaxis and the other after the end of it. Conclusion: The prevalence of the cytomegalovirus disease is slightly lower than in other studies. Moreover, the cytomegalovirus disease can remit with a prompt diagnosis and the proper medical treatment.

Key words: Heart transplantation, cytomegalovirus, serology, prevalence.

Palabras clave: Trasplante de corazón, citomegalovirus, serología, prevalencia.

\section{Introducción}

$\mathrm{E}$ 1 trasplante cardiaco es el procedimiento quirúrgico de elección ante la falla de la terapia médica o quirúrgica para mantener las funciones cardiacas ${ }^{1}$. A pesar de las medidas preventivas que se efectúan para evitar las infecciones, el desarrollo de éstas por bacterias, virus u hongos genera una importante morbilidad y mortalidad en los pacientes receptores de trasplantes del corazón ${ }^{2}$; un patógeno viral de consideración en los pacientes que reciben trasplante de órganos sólidos es citomegalovirus (CMV) ${ }^{3,4}$. La infección por CMV se puede establecer mediante la presencia de replicación viral, detección de las proteínas virales (antigenemia) y su material genético o el crecimiento in vitro del virus en pacientes sin alguna sintomatología. La enfermedad, a su vez, es la manifestación de síntomas y signos atribuibles a la infección por CMV; se puede dividir en síndrome viral, donde se confirma la replicación viral por pruebas de laboratorio; y la enfermedad invasora, que evidencia síntomas específicos de la afectación de un órgano con la demostración histológica del daño ${ }^{5}$.

Citomegalovirus continua siendo el agente que produce la mayor cantidad de infecciones en pacientes receptores de trasplante de órganos sólidos ${ }^{6}$; específicamente en el trasplante cardiaco, CMV es la mayor causa de infección después del procedimiento quirúrgico ${ }^{7}$. Se reporta que la incidencia de enfermedad por CMV en trasplante cardiaco varía entre 7 y $9 \%$, donde el antecedente de donante seropositivo y receptor negativo determina alto riesgo de aparición de la infección, riesgo que ocurre y se mantiene en el período post-trasplante a pesar de tres meses de profilaxis antiviral ${ }^{8-10}$.

Se han descrito dos estrategias preventivas contra la infección por CMV, una de las cuales consiste en la administración profiláctica, durante tres a seis meses, de ganciclovir (parenteral) o valganciclovir (oral); la segunda estrategia consiste en la monitorización periódica de la carga viral de CMV en el período post-trasplante y la administración de fármacos antivirales una vez que se manifiesta la infección (terapia anticipada o preemptive therapy en inglés $)^{11}$. La primera terapia está asociada a menor viremia, expone al paciente a un riesgo mayor de aparición tardía de la infección y mayores efectos adversos que la terapia anticipada ${ }^{2}$. La "International Socierty of Heart and Lung Transplantation" (ISHLT) reporta que la profilaxis para evitar la infección consiste en la administración de ganciclovir intravenoso, seguido de valganciclovir oral una vez estabilizado el paciente ${ }^{13}$. Si el paciente desarrolla enfermedad por CMV, el tratamiento
Instituto Nacional

Cardiovascular. Lima-Perú. Servicio de Infectología (CR). Universidad Científica del Sur (UCSUR). Lima-Perú. Estudiante de Medicina Humana (GDLCK, BVV). Sociedad Científica de Estudiantes de Medicina Humana SCIEM UCSUR. Lima-Perú (GDLCK)

El artículo fue financiado por los propios autores. Además, el proyecto fue aprobado por el Comité Institucional del Instituto Nacional Cardiovascular. Ninguno de los autores reporta tener alguna clase de conflicto de intereses para el desarrollo del presente estudio.

Recibido: 14 de julio de 2016 Aceptado: 29 de septiembre de 2016

Correspondencia a: Christian Rojas Contreras christeorc@gmail.com 
se realiza con los mismos fármacos; y si se desarrolla resistencia a éstos se utilizará foscarnet, agente de segunda línea para el tratamiento del $\mathrm{CMV}^{3}$.

A pesar que el trasplante cardiaco es un procedimiento financiado por el sistema de salud peruano, la demanda de personas que precisan un trasplante de órganos no cubre la oferta de los servicios de salud ${ }^{14,15}$. Sobre la situación actual del trasplante cardiaco en Perú, el reporte del Consulado Europeo y la Organización Nacional de Trasplante indica que 17 pacientes ingresaron por primera vez a la lista en el último año, lo que generó un acumulado de 22 pacientes en esta lista, donde dos de ellos fallecieron a la espera del trasplante ${ }^{15}$.

El propósito de este estudio fue describir las características de la enfermedad por CMV en pacientes que recibieron trasplante de corazón, revisar la presentación clínica y el tratamiento farmacológico de los pacientes que desarrollaron la enfermedad por CMV, en un centro de trasplante cardiaco en Perú.

\section{Materiales y Métodos}

\section{Diseño de estudio y pacientes}

Estudio retrospectivo, descriptivo, realizado en el Instituto Nacional Cardiovascular (INCOR), Lima, Perú, con aprobación del Comité de Ética institucional. Cabe mencionar que el INCOR es el único establecimiento acreditado para la realización de trasplante cardiaco en Perú ${ }^{16}$. Se incluyó a los 35 pacientes que recibieron trasplante de corazón (todos), entre los años 2010 y 2015. La información se obtuvo a través de la revisión de la ficha clínica de los receptores, no se tuvo disponibilidad a la ficha clínica ni estado serológico para CMV en los donantes.

La terapia de inducción fue basiliximab y metilprednisolona, en la terapia de mantención se utilizó micofenolato mofetil, tracolimus y corticoides. En la profilaxis para $\mathrm{CMV}$, se empleó ganciclovir $5 \mathrm{mg} / \mathrm{kg}$ cada $12 \mathrm{~h}$ durante una semana y posteriormente valganciclovir $900 \mathrm{mg}$ cada $24 \mathrm{~h}$ durante tres meses.

\section{Variables sociodemográficas y clínicas}

Se analizaron las co-morbilidades y exámenes de laboratorio previos a la cirugía; estado nutricional pre y post-trasplante cardiaco, mediante la medición de la albúmina sérica. Se registraron las características sociodemográficas de los pacientes que presentaron enfermedad por CMV. Para detectar la infección por CMV se utilizó la reacción de polimerasa en cadena (RPC).

\section{Análisis estadístico}

En el análisis de las variables socio-demográficas y clínicas de tipo cuantitativas se emplearon la media y la desviación estándar. Para las variables cualitativas se usaron las frecuencias y las proporciones. Se usó el programa estadístico SPSS versión 22.

\section{Resultados}

La edad promedio fue 40 años y $63 \%$ eran varones. Seis pacientes $(17 \%)$ presentaron hipertensión arterial y nueve pacientes $(25,4 \%)$ diabetes mellitus; ninguno de ellos desarrolló enfermedad por CMV. El colesterol total de la población fue, en promedio 149,44 $\pm 49,60$ $\mathrm{mg} / \mathrm{dL}$; a su vez, los valores de HDL fueron de $36,10 \pm$ $16,19 \mathrm{mg} / \mathrm{dL}$, mientras que los niveles de LDL, VLDL y triglicéridos se encontraron en rangos normales. El índice de masa corporal (IMC), en el período pre quirúrgico tuvo una media de $23 \pm 4,23 ; 24$ pacientes $(69 \%)$ tuvieron un IMC mayor o igual a 25; 10 pacientes presentaron IMC inferior a 20, dos de ellos desarrollaron la enfermedad por CMV. Después de la cirugía, uno de ellos presentó IMC normal.

Hubo un promedio de 2,00 $\pm 1,94$ infecciones post trasplante por paciente. De los 10 pacientes hospitalizados previamente a la cirugía, uno $(7,14 \%)$ desarrolló la enfermedad por CMV.

En relación a la serología IgG para CMV, se observó que de 35 pacientes, 68,6\% presentaba $\operatorname{IgG}(+)$ pre trasplante y ninguno de ellos desarrolló la enfermedad, mientras que dos de los 11 pacientes (18,2\%) IgG (-) pre-trasplante, la desarrollaron. La prevalencia global de enfermedad por CMV fue de 5,7\%.

La depuración de creatinina en los pacientes que presentaron enfermedad por CMV correspondió a insuficiencia renal aguda (IRA) etapa 1 . Uno de los dos pacientes con enfermedad por CMV presentó concentraciones bajas de albúmina $(3,24 \mathrm{~g} / \mathrm{dL})$.

En la Tabla 1 se muestran las características demográficas de los dos pacientes que desarrollaron enfermedad por CMV. El primer paciente fue un varón de 41 años cuya indicación de trasplante fue una cardiomiopatía dilatada. La enfermedad por CMV se inició en el día 68 posttrasplante cardiaco y duró 21 días. Se presentó con fiebre, insuficiencia respiratoria e insuficiencia renal aguda, una elevación de creatinina sérica de 1,22 hasta $2 \mathrm{mg} / \mathrm{dL}$; la carga viral más elevada fue de 1.880 copias $/ \mathrm{mL}$ al momento del diagnóstico. La enfermedad remitió con ganciclovir endovenoso y luego continuó con valganciclovir oral por dos meses, luego de disminuir la carga a indetectable. El segundo paciente, un niño de 11 años, receptor de trasplante, también por miocadiopatía dilatada, manifestó la enfermedad a los 137 días post-trasplante cardiaco, la que duró un año. Presentó síntomas inespecíficos, fiebre $38,6^{\circ} \mathrm{C}$, la carga viral más elevada fue de 399.704 copias/ $\mathrm{mL}$ en el día 157 post-trasplante cardiaco y se trató con 


\begin{tabular}{lll}
$\begin{array}{l}\text { Tabla 1. Detalle clínico de los pacientes que desarrollaron enfermedad por citomegalovirus post trasplante cardiaco. INCOR, } \\
\text { Lima, Perú. 2010-2015 }\end{array}$ & Paciente 1 \\
\hline & & Paciente 2 \\
\hline Características demográficas & 41 & 11 \\
Edad al Tx cardiaco & Masculino & Masculino \\
Sexo & Congénita & Congénita \\
Etiología de la falla cardiaca & Síl & Sí \\
Vivo & & \\
Características clínicas & 68 & 137 \\
Días post-Tx cardiaco & 26 & 25 \\
Tiempo de profilaxis con ganciclovir (días) & 3 & 3 \\
Tiempo de profilaxis con valganciclovir (meses) & 21 & 327 \\
Duración de la infección/ enfermedad (días) & Fiebre & Úlceras orales \\
Clínica & Insuficiencia respiratoria & Dolor abdominal difuso \\
& Insuficiencia renal aguda & Deposiciones líquidas \\
& Infiltración a la médula ósea & Sepsis \\
Carga viral más elevada (copias/mL) & 1.880 & 399.704 \\
Tratamiento-fármaco (días) & Ganciclovir (25) & Foscarnet (90) \\
& & Ganciclovir (175) \\
\hline Tx: trasplante. & & \\
& &
\end{tabular}

foscarnet durante 90 días por resistencia al tratamiento con ganciclovir. Ambos pacientes mejoraron y están vivos al momento de redactar esta publicación.

\section{Discusión}

La infección por CMV en pacientes receptores de trasplante cardiaco, continúa siendo frecuente y produciendo enfermedad por $\mathrm{CMV}^{9}$. La literatura al respecto en población peruana es escasa, por lo que esta visión retrospectiva pretende describir las características demográficas, clínicas, y serológicas de los pacientes que presentaron enfermedad por CMV en un centro donde se realiza trasplante cardiaco.

Entre 35 y $80 \%$ de los pacientes que son sometidos a trasplante de órganos sólidos desarrollan infección por $\mathrm{CMV}^{8,17,18}$. Boland y cols. ${ }^{19}$, describieron la infección en $50 \%$ de los receptores de trasplante cardiaco. Un reporte más reciente de Santos y cols. ${ }^{9}$, afirma que la enfermedad por CMV sólo se presenta en 7,5 a 9\% de los infectados. Gupta y cols. ${ }^{10}$, reportaron que 6 de 7 pacientes que recibieran trasplante cardiaco presentaron enfermedad por CMV. En la presente experiencia, 68,6\% de los pacientes presentaban serología positiva a CMV; no obstante, sólo 2 de 35 pacientes $(5,7 \%)$ desarrollaron la enfermedad por CMV, a diferencia de $18,2 \%$ con serología negativa pre trasplante, resultados que asemejan a lo reportado en la literatura médica.

En nuestro centro de trasplante, todos los pacientes reciben profilaxis estandarizada post-trasplante con ganciclovir endovenoso durante una semana y posteriormente valganciclovir oral por tres meses, independientemente del status serológico para CMV. Con esta medida la probabilidad de infección, enfermedad y mortalidad por CMV disminuyó significativamente ${ }^{17}$. Julian y cols..$^{20}$, reportaron que al administrar una dosis baja de valganciclovir por tres a seis meses, $22 \%$ de los receptores seronegativos y $2,7 \%$ de los receptores seropositivos desarrollaron infección por $\mathrm{CMV}$, hallazgos similares a nuestra población. En este reporte la dosis de valganciclovir fue de $900 \mathrm{mg}$ cada $24 \mathrm{~h}$, por tres meses, mientras que ganciclovir es empleado como profilaxis estándar adicional.

En relación al IMC pre quirúrgico, en ocho pacientes fue menor a 20; de ellos sólo dos desarrollaron enfermedad por CMV. Se reporta que un IMC bajo también está asociado a diversas condiciones; por ejemplo, Chen y cols. ${ }^{21}$, demostraron que los receptores de trasplante cardiaco con menores niveles de IMC, tenían una mayor leucopenia $(p=0,008)$. Además, Weber y cols. ${ }^{22}$, establecieron que un IMC menor a 20 está asociado a una menor mortalidad en personas entre 18 y 35 años $(p<0,05)$. Sin embargo, en personas de mayor edad, la mortalidad fue superior con un IMC menor a 20 , sin significancia 
estadística. Estos resultados indican que la variable IMC está involucrada en diversas condiciones clínicas, las que afectan negativamente el estado clínico de los pacientes, por lo que su análisis debe de ser sistemático, con el fin de identificar valores alterados y tomar medidas para prevenir futuros eventos adversos.

Sobre la serología, Delgado y cols. ${ }^{23}$, describen que ser un receptor seronegativo para $\mathrm{CMV}$, predispone a la infección y enfermedad, lo que fue confirmado en esta casuística, en que dos pacientes con serología negativa para CMV desarrollaron infección y luego enfermedad post-trasplante cardiaco.

Según un meta análisis ${ }^{24}$, la profilaxis con antivirales disminuye el riesgo de enfermedad por CMV (RR: 0,42, 95\%: CI 0,34-0,52) y la infección por CMV (RR: 0,61, 95\%: CI 0,48-0,77). La presentación de la enfermedad por CMV ocurre con una mayor frecuencia después de los 100 días'; sin embargo, un tercio de los pacientes post trasplante de órganos sólidos manifiestan la infección antes del año ${ }^{25}$. Todos nuestros pacientes recibieron la quimioprofilaxis; no obstante, dos de ellos desarrollaron la enfermedad después de los 100 días. La infección puede aparecer entre el segundo cuarto, o sexto mes post-trasplante ${ }^{26}$, lo que se corrobora con la historia de los pacientes.

El primer paciente presentó la enfermedad mientras se encontraba en profilaxis con valganciclovir, al detectar la enfermedad, se trató con ganciclovir y remitió. El segundo, presentó resistencia clínica y virológica al tratamiento con ganciclovir y valganciclovir, por lo que recibió foscarnet, según se recomienda en la literatura científica $^{3,4,27}$. El fármaco es un análogo orgánico de pirofosfato inorgánico, que se une a la $\mathrm{ADN}$ polimerasa ${ }^{28}$, con lo que las cargas virales del paciente fueron indetectables. La larga duración de la infección que presentó el paciente fue debido a la demora de la obtención del fármaco dado que no estaba disponible en el Perú.

Entre las manifestaciones clínicas de la enfermedad por CMV se encuentra comúnmente la gastrointestinal en pacientes con posterioridad a trasplante de órganos sólidos ${ }^{29,30}$; similar situación se presenta en los trasplantes de corazón ${ }^{31}$. En nuestra serie, un paciente de los dos que desarrollaron la enfermedad tuvo un compromiso gastrointestinal manifestado por dolor abdominal difuso y deposiciones acuosas. Por otro lado, también hubo compromiso de la médula ósea. Ambas situaciones pueden interpretarse como una afectación tisular por parte del CMV aunque no se objetivó la presencia del virus en estos tejidos.

El presente reporte tiene ciertas limitaciones; en primer lugar, el número de pacientes es escaso, impidiendo una extrapolación certera de los resultados. También se desconoce la información demográfica, y serológica de CMV de los donantes, por lo que esto restringió un análisis más exhaustivo en esta población en específico. No se contó con las cargas virales de todos los pacientes, sólo se cuantificó a los que afectó clínicamente la enfermedad. No obstante, los resultados encontrados contribuyen al conocimiento de esta población. Ante lo expuesto se recomienda aumentar la población a estudiar y lograr mayor información del estado serológico de los donantes.

$\mathrm{La}$ infección y enfermedad por CMV permanecen como una causa de morbilidad en la población estudiada, en quienes se realizó un tratamiento según recomendaciones actuales de las guías clínicas, lo que influye en la recuperación y sobrevida de los pacientes ${ }^{32}$. Además, se rescata que la incidencia de la enfermedad por CMV fue ligeramente menor a comparación de otros estudios. Es de consideración mencionar que, a pesar de características diferentes de ambos pacientes, se logró controlar la enfermedad por CMV.

Agradecimientos. Se agradece al equipo de trasplante cardiaco del INCOR por su colaboración en todo momento, la cual fue necesaria para la elaboración y culminación de la presente investigación.

\section{Resumen}

Introducción: El trasplante cardiaco es el tratamiento de elección ante la falla cardiaca refractaria a la terapia médica o quirúrgica. En base a ello, la enfermedad por citomegalovirus (CMV) es una importante complicación infecciosa post-trasplante de corazón. Objetivos: Describir la prevalencia y las características clínicas de los pacientes que desarrollaron enfermedad por CMV posttrasplante de corazón. Materiales y Métodos: Se realizó un estudio retrospectivo y descriptivo, donde se incluyó a los 35 pacientes que recibieron trasplante de corazón en el Instituto Nacional Cardiovascular entre el período 2010-2015. La información se obtuvo mediante la revisión de historias clínicas. Se analizaron las variables demográficas y clínicas relevantes de los casos con enfermedad por CMV. Resultados: La edad media de la población fue de 39,49 $\pm 15,07$ años, siendo la mayoría de sexo masculino (63\%). La prevalencia de la enfermedad por CMV fue de 5,7\%,-dos pacientes-, ambos con serología negativa para CMV previa al trasplante. Uno de ellos presentó la enfermedad antes de terminar la profilaxis con valganciclovir y el otro luego del cese de la misma. Conclusión: La prevalencia de la enfermedad por CMV es ligeramente menor que en otros estudios. Asimismo, ésta puede remitir con un pronto diagnóstico y el adecuado tratamiento médico. 


\section{Referencias bibliográficas}

1.- Hullin R. Heart transplantation: current practice and outlook to the future. Swiss Med Wkly 2014; 144: w13977.

2.- Ubilla M, Mastrobuoni S, Martín Arnau A, Cordero A, Alegría E, Gavira J J, et al. Trasplante cardíaco. An Sist San Navar 2006; 29: 63-78.

3.- Kotton C N, Kumar D, Caliendo A M, Asberg A, Chou S, Danziger-Isakov L, et al. Updated international consensus guidelines on the management of cytomegalovirus in solidorgan transplantation. Transplantation 2013; 96 (4): 333-60.

4.- de la Torre-Cisneros J, Farinas M C, Caston J J, Aguado J M, Cantisan S, Carratala J, et al. GESITRA-SEIMC/REIPI recommendations for the management of cytomegalovirus infection in solid-organ transplant patients. Enferm Infecc Microbiol Clin 2011; 29 (10): 735-58.

5.- Azevedo L S, Pierrotti L C, Abdala E, Costa S F, Strabelli T M, Campos S V, et al. Cytomegalovirus infection in transplant recipients. Clinics (Sao Paulo, Brazil) 2015; 70 (7): 515-23.

6.- Kalil A C, Levitsky J, Lyden E, Stoner J, Freifeld A G. Meta-analysis: The efficacy of strategies to prevent organ disease by cytomegalovirus in solid organ transplant recipients. Ann Intern Med 2005; 143 (12): 870-80.

7.- Abate D, Fiscon M, Saldan A, Cofano S, Mengoli C, Sgarabotto D, et al. Human cytomegalovirus-specific T-cell immune reconstitution in preemptively treated heart transplant recipients identifies subjects at critical risk for infection. J Clin Microbiol 2012; 50 (6): 1974-80.

8.- Méndez-Eirin E, Paniagua-Martin M J, Marzoa-Rivas R, Barge-Caballero E, Grille-Cancela Z, Canizares A, et al. Cumulative incidence of cytomegalovirus infection and disease after heart transplantation in the last decade: effect of preemptive therapy. Transplant Proc 2012; 44 (9): 2660-2.

9.- Santos C A, Brennan D C, Fraser V J, Olsen M A. Incidence, risk factors, and outcomes of delayed-onset cytomegalovirus disease in a large, retrospective cohort of heart transplant recipients. Transplant Proc 2014; 46 (10): 3585-92

10.- Gupta S, Mitchell J D, Markham D W, Mammen P P, Patel P C, Kaiser P, et al. High incidence of cytomegalovirus disease in
D+/R- heart transplant recipients shortly after completion of 3 months of valganciclovir prophylaxis. J Heart Lung Transplant 2008; 27 (5): 536-9.

11.- Manuel O, Kralidis G, Mueller N J, Hirsch H H, Garzoni C, van Delden C, et al. Impact of antiviral preventive strategies on the incidence and outcomes of cytomegalovirus disease in solid organ transplant recipients. Am J Transplant 2013; 13 (9): 2402-10.

12.- Florescu D F, Qiu F, Schmidt C M, Kalil A C. A direct and indirect comparison meta-analysis on the efficacy of cytomegalovirus preventive strategies in solid organ transplant. Clin Infect Dis 2014; 58 (6): 785-803.

13.- Costanzo M R, Dipchand A, Starling R, Anderson A, Chan M, Desai S, et al. The International Society of Heart and Lung Transplantation Guidelines for the care of heart transplant recipients. J Heart Lung Transplant 2010; 29 (8): 914-56.

14.- Ministerio de Salud. Aprueban Reglamento de la Ley $N^{\circ} 29.471$, Ley que promueve la obtención, la donación y el trasplante de órganos o tejidos humanos. Lima: El Peruano; 2010.

15.- European Directorate for the Quality of Medicines \& Health Care. Newsletter Transplant. Spain: EDQM/ONT, 2015.

16.- Ministerio de Salud. Buscador de Normal Legales [Internet]. MINSA; 2016 [cited 2016 19/09]. Available from: http://www.minsa.gob. pe/transparencia/dge_normas.asp.

17.- Roman A, Manito N, Campistol J M, Cuervas-Mons V, Almenar L, Arias M, et al. The impact of the prevention strategies on the indirect effects of CMV infection in solid organ transplant recipients. Transplant Rev (Orlando, Fla) 2014; 28 (2): 84-91.

18.- Niubo J, Pérez J L, Martínez-Lacasa J T, García A, Roca J, Fabregat J, et al. Association of quantitative cytomegalovirus antigenemia with symptomatic infection in solid organ transplant patients. Diag Microbiol Infect Dis 1996; 24 (1): 19-24

19.- Boland G J, Ververs C, Hene R J, Jambroes G, Donckerwolcke R A, de Gast G C. Early detection of primary cytomegalovirus infection after heart and kidney transplantation and the influence of hyperimmune globulin prophylaxis. Transplant Intern 1993; 6 (1): 34-8.

20.- Julian K G, Shattahi E, Burg J E, Boehmer J P. Low-dose valganciclovir for cytomegalovirus prophylaxis in heart transplant recipients. Transplant Proc 2013; 45 (9): 3414-7.
21.- Chen I M, Chang H H, Hsu C P, Lai S T, Hsieh Y C, Shih C C. Correlation between body mass index and leucopenia after administration of valganciclovir for cytomegalovirus infection in chinese cardiac recipients. Circulation J 2007; 71 (6): 968-72.

22.- Weber D J, Didolkar P, Gracon A, Hellman Y, Hadi M A, Malik A, et al. The role of recipient $\mathrm{BMI}$ and age on survival after heart transplantation. J Heart Lung Transplant 2014; 33 (4): S91.

23.- Delgado J F, Manito N, Almenar L, CrespoLeiro M, Roig E, Segovia J, et al. Risk factors associated with cytomegalovirus infection in heart transplant patients: a prospective, epidemiological study. Transplant Infect Dis 2011; 13 (2): 136-44.

24.- Hodson E M, Ladhani M, Webster A C, Strippoli G F, Craig J C. Antiviral medications for preventing cytomegalovirus disease in solid organ transplant recipients. The Cochrane database of systematic reviews. 2013; 2: $\mathrm{Cd} 003774$

25.- Paya C, Humar A, Domínguez E, Washburn K, Blumberg E, Alexander B, et al. Efficacy and safety of valganciclovir vs. oral ganciclovir for prevention of cytomegalovirus disease in solid organ transplant recipients. Am J Transplant 2004; 4 (4): 611-20.

26.- Palomo J. Trasplante Cardiaco. Madrid 1996.

27.- Lumbreras C G J, Cisneros J M, Muñoz P. Infecciones en el paciente trasplantado. Protocolos SEIMC. 2005.

28.- Balfour H H. Antiviral drugs. N Engl J Med 1999; 340 (16): 1255-68.

29.- Blyth D, Lee I, Sims K D, Gasink L B, Barton TD, Van Deerlin V M, et al. Risk factors and clinical outcomes of cytomegalovirus disease occurring more than one year post solid organ transplantation. Transplant Infect Dis 2012; 14 (2): 149-55.

30.- McCarty T P, Lee R A, Herfel B M, Pappas P G. Cytomegalovirus appendicitis in solid organ transplant patients, two cases and a review. J Clin Virol 2015; 66: 48-50.

31.- Kaplan C S, Petersen E A, Icenogle T B, Copeland J G, Villar H V, Sampliner R, et al. Gastrointestinal cytomegalovirus infection in heart and heart-lung transplant recipients. Arch Intern Med 1989; 149 (9): 2095-100.

32.- Van Bakel A B, Brown R N, Nikolaidis L A, Heroux A, Law K, Naftel D C. Variations in institutional staffing and clinical practice are predictive of center-specific 1-year survival post-transplant. J Heart Lung Transplant 2013; 32 (12): 1196-204 